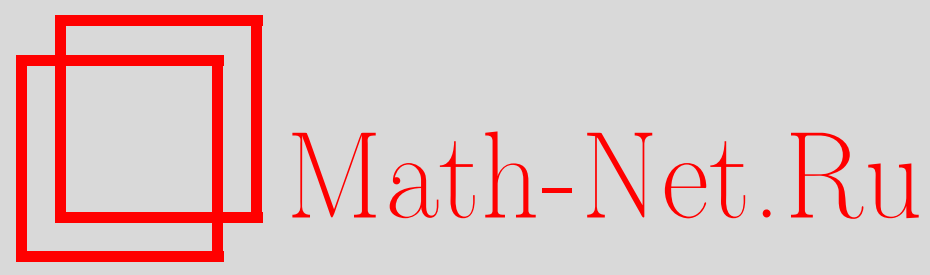

Ф. Г. Авхадиев, Д. В. Маклаков, Новые уравнения типа свертки, получаемые заменой интеграла на максимум, $M a-$ тем. заметки, 2002, том 71, выпуск 1, 18-26

DOI: https://doi.org/10.4213/mzm324

Использование Общероссийского математического портала Math-Net.Ru подразумевает, что вы прочитали и согласны с пользовательским соглашением http://www . mathnet.ru/rus/agreement

Параметры загрузки:

IP : 54.198 .55 .26

26 апреля 2023 г., 02:51:57

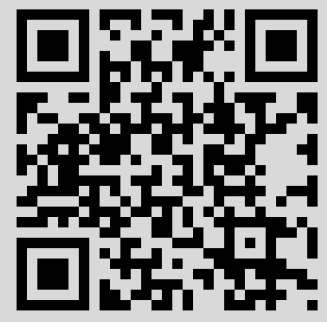




\title{
НОВЫЕ УРАВНЕНИЯ ТИПА СВЕРТКИ, ПОЛУЧАЕМЫЕ ЗАМЕНОЙ ИНТЕГРАЛА НА МАКСИМУМ
}

\author{
Ф. Г. Авхадиев, Д. В. Маклаков
}

Для неотрицательных, непрерьвных, $\pi$-периодических функций $g(\gamma)$ исследуется нелинейное уравнение

$$
\max _{\gamma \in \mathbb{R}} g(\gamma)|\cos (\gamma-\alpha)|=f(\alpha)
$$

где $f(\alpha)$ - заданная, $g(\gamma)$ - искомая функции. Это уравнение возникло при решении прикладной задачи о построении гидропрофиля по заданной кавитационной диаграмме. Получены необходимые и достаточные условия разрешимости, явное описание множества всех решений и теорема сравнения при изменениях правой части. Указаны возможные пути обобщения.

Библиографояи: 5 названий.

1. Введение. В статьях [1]-[3] нами дано решение прикладной задачи о построении гидропрофиля по заданной кавитационной диаграмме. В этой задаче возникло новое уравнение типа свертки

$$
\max _{\gamma \in \mathbb{R}} g(\gamma)|\cos (\gamma-\alpha)|=f(\alpha)
$$

где $f(\alpha)$ - заданная, $g(\gamma)$ - искомая функции.

Уравнение (1) является нелинейным и отличается от обычных уравнений типа свертки заменой интеграла на максимум по переменной интегрирования. Отметим, что для неотрицательных $g(\gamma)$ уравнение (1) можно рассматривать как предельньй случай при $p \rightarrow+\infty$ уравнения вида

$$
\left(\int|g(\gamma) \cos (\gamma-\alpha)|^{p} d \gamma\right)^{1 / p}=f(\alpha), \quad \alpha \in \mathbb{R}
$$

В настоящей работе мы исследуем разрешимость уравнения (1) в классе $\pi$-периодических, непрерьвных функций при наиболее широких предположениях относительно правой части, получаем явную формулу обращения и описание множества всех решений, а также доказываем теорему о сравнении решений при изменениях правой части. В заключительной части статьи мы указываем на возможность обобщения уравнения (1) на случай ядра, отличного от $|\cos (\gamma-\alpha)|$.

Работа выполнена при финансовой поддержке Российского фонда фундаментальных исследований, гранты № 99-01-00366, № 99-01-00169, № 99-01-00173. 
2. Основные результаты. Пусть $f(\alpha)$ определена на всей действительной оси $\mathbb{R}$. Решение уравнения (1) будем искать в следующем классе:

$$
G=\{g \in C(\mathbb{R}): g(\gamma) \geqslant 0, g(\gamma+\pi)=g(\gamma), \gamma \in \mathbb{R}\} \backslash\{0\}
$$

т.е. $G$ - множество непрерывных, неотрицательных, $\pi$-периодических функций, не обращающихся тождественно в нуль.

Обозначим

$$
T=\{f \in G: f(\alpha)>0, f(\alpha) \text { тригонометрически вьпукла }\} \text {, }
$$

т.е. $T$ - подмножество $G$, состоящее из строго положительных, тригонометрически выпуклых функций.

Напомним (см. [4], [5]), что функция $f(\alpha)$ назьвается тригонометрически выпуклой (порядка 1), если для любых $\alpha_{1}$ и $\alpha_{2}, 0<\alpha_{2}-\alpha_{1}<\pi$, выполняется неравенство

$$
f(\alpha) \leqslant H(\alpha), \quad \alpha_{1}<\alpha<\alpha_{2},
$$

где

$$
H(\alpha)=\frac{f\left(\alpha_{1}\right) \sin \left(\alpha_{2}-\alpha\right)+f\left(\alpha_{2}\right) \sin \left(\alpha-\alpha_{1}\right)}{\sin \left(\alpha_{2}-\alpha_{1}\right)} .
$$

Геометрически неравенство (3) означает, что график функции $y=f(\alpha)$ над отрезком $\left[\alpha_{1}, \alpha_{2}\right]$ лежит не вьше “тригонометрической хорды", определяемой уравнением вида $y=H(\alpha)=a \cos \alpha+b \sin \alpha$ и условиями $H\left(\alpha_{1}\right)=f\left(\alpha_{1}\right), H\left(\alpha_{2}\right)=f\left(\alpha_{2}\right)$.

Справедливы следующие утверждения.

Теорема 1. Для разрешимости уравнения (1) в классе $G$ необходимо и достаточно, чтобь $f \in T$. Если $f \in T$, то функиия

$$
g_{m}(\gamma ; f)=\min _{\alpha \in \mathbb{R}} \frac{f(\alpha)}{|\cos (\gamma-\alpha)|}
$$

принадлежит классу $G$ и является решением уравнения (1).

Напомним, что если $f \in T$, то всюду существуют левая и правая производные $f^{\prime}(\alpha \pm 0)$, причем $f^{\prime}(\alpha-0) \leqslant f^{\prime}(\alpha+0)$, а точки разрьва самой производной $f^{\prime}(\alpha)$ составляют не более чем счетное множество (см., например, [4]). Обозначим

$$
q(\alpha)=\alpha+\operatorname{arctg} \frac{f^{\prime}(\alpha)}{f(\alpha)}
$$

Условие единственности решения (5) и множество всех решений описывает следующая теорема 2. 
ТЕорема 2. Формула обращения (5) дает единственное решение уравнения (1) в классе $G$ тогда и только тогда, когда $f(\alpha)$ имеет непрерывную всюду производную. Если $f^{\prime}(\alpha)$ имеет точки разрыва, то любая функиия $g \in G$ будет решением уравнения (1) тогда и только тогда, когда она удовлетворяет условиям

$$
\begin{array}{ll}
g(\gamma)=g_{m}(\gamma ; f), & \gamma \in \mathbb{R} \backslash X_{f}, \\
g(\gamma) \leqslant g_{m}(\gamma ; f), & \gamma \in X_{f},
\end{array}
$$

$2 \partial e$

$$
X_{f}=\bigcup_{\alpha \in I}(q(\alpha-0), q(\alpha+0)),
$$

причем обвединение берется по множеству I точек разрыва $f^{\prime}(\alpha)$.

Таким образом, решение $g_{m}(\gamma ; f)$ является максимальным среди всех решений уравнения (1).

Из формулы (5) непосредственно следует, что $g_{m}(\gamma ; f) \geqslant g_{m}\left(\gamma ; f^{*}\right)$, если $f(\alpha) \geqslant f^{*}(\alpha)$ для всех $\alpha \in \mathbb{R}$. Если же $f(\alpha) \geqslant f^{*}(\alpha)$ лиш на некотором отрезке $\left[\alpha_{1}, \alpha_{2}\right]$ и $0<\alpha_{2}-\alpha_{1}$ $<\pi$, то сравнение максимальных решений становится нетривиальной задачей. Тем не менее, сравнение оказьвается возможным и в этом случае.

Tеорема 3. Пусть $f u f^{*} \in T, q(\alpha)=\alpha+\operatorname{arctg}\left[f^{\prime}(\alpha) / f(\alpha)\right] . \operatorname{Ec} \Omega u f(\alpha) \geqslant f^{*}(\alpha)$ npu $\alpha_{1} \leqslant \alpha \leqslant \alpha_{2}$, mo

$$
g_{m}(\gamma ; f) \geqslant g_{m}\left(\gamma ; f^{*}\right)
$$

для всех $\gamma \in\left[q\left(\alpha_{1}-0\right), q\left(\alpha_{2}+0\right)\right]$.

3. Доказательство теорем 1-3. Нам потребуется ряд свойств функции $q(\alpha)$, определяемой соотношением (6). Из известных свойств тригонометрически вьпуклых функций непосредственно получаем: если $f \in T$, то

a) $q(\alpha)$ определена и непрерьвна всюду в $\mathbb{R}$, за исключением разве лиш счетного множества точек $I$;

б) в любой точке $\alpha \in I$ существуют конечные пределы справа $q(\alpha+0)$ и слева $q(\alpha-0)$, причем $q(\alpha-0) \leqslant q(\alpha+0)$, функция $q(\alpha+0)$ непрерьвна справа, а функция $q(\alpha-0)$ непрерывна слева.

Докажем следующее свойство положительных тригонометрически выпуклых функций.

Лемма 1. Пусть $f \in T$. Тогда функиия $q(\alpha)$ является неубывающей.

ДокАЗАТЕЛЬСтво. Нам достаточно показать, что для любых $\alpha_{1}$ и $\alpha_{2} \in \mathbb{R}, 0<$ $\alpha_{2}-\alpha_{1}=\Delta<\pi / 2$, выполняется неравенство

$$
q\left(\alpha_{2}-0\right) \geqslant q\left(\alpha_{1}+0\right)
$$

Через точки $\left(\alpha_{1}, f\left(\alpha_{1}\right)\right)$ и $\left(\alpha_{2}, f\left(\alpha_{2}\right)\right)$ проведем тригонометрическую хорду $H(\alpha)$, задаваемую уравнением (4). Так как производные $f^{\prime}\left(\alpha_{1}+0\right)$ и $f^{\prime}\left(\alpha_{2}-0\right)$ существуют, то в силу (3) имеем

$$
H^{\prime}\left(\alpha_{1}\right) \geqslant f^{\prime}\left(\alpha_{1}+0\right), \quad H^{\prime}\left(\alpha_{2}\right) \leqslant f^{\prime}\left(\alpha_{2}-0\right) \text {. }
$$


Отсюда вытекает, что

$$
\begin{aligned}
& f^{\prime}\left(\alpha_{1}+0\right) \leqslant-f\left(\alpha_{1}\right) \operatorname{ctg} \Delta+\frac{f\left(\alpha_{2}\right)}{\sin \Delta}, \\
& f^{\prime}\left(\alpha_{2}-0\right) \geqslant-\frac{f\left(\alpha_{1}\right)}{\sin \Delta}+f\left(\alpha_{2}\right) \operatorname{ctg} \Delta .
\end{aligned}
$$

Доказьваемое неравенство (9) эквивалентно соотношению

$$
\alpha_{2}-\alpha_{1} \geqslant \operatorname{arctg} \frac{f^{\prime}\left(\alpha_{1}+0\right)}{f\left(\alpha_{1}\right)}-\operatorname{arctg} \frac{f^{\prime}\left(\alpha_{2}-0\right)}{f\left(\alpha_{2}\right)} .
$$

Если правая часть неравенства (12) меньше или равна нулю, то доказьвать нечего. Поэтому предположим, что

$$
\frac{f^{\prime}\left(\alpha_{1}+0\right)}{f\left(\alpha_{1}\right)}>\frac{f^{\prime}\left(\alpha_{2}-0\right)}{f\left(\alpha_{2}\right)} .
$$

Тогда правая часть $(12)$ лежит в интервале $(0, \pi)$, и мы можем взять котангенсы от обеих частей. С учетом (13) доказываемое неравенство примет вид

$$
\operatorname{ctg}\left(\alpha_{2}-\alpha_{1}\right)\left[\frac{f^{\prime}\left(\alpha_{1}+0\right)}{f\left(\alpha_{1}\right)}-\frac{f^{\prime}\left(\alpha_{2}-0\right)}{f\left(\alpha_{2}\right)}\right] \leqslant 1+\frac{f^{\prime}\left(\alpha_{1}+0\right) f^{\prime}\left(\alpha_{2}-0\right)}{f\left(\alpha_{1}\right) f\left(\alpha_{2}\right)} .
$$

В силу строгой положительности $f(\alpha)$ из (10) и (11) получаем

$$
\begin{aligned}
\frac{f^{\prime}\left(\alpha_{1}+0\right)}{f\left(\alpha_{1}\right)}+\operatorname{ctg} \Delta & \leqslant \frac{f\left(\alpha_{2}\right)}{f\left(\alpha_{1}\right)} \cdot \frac{1}{\sin \Delta}, \\
-\frac{f^{\prime}\left(\alpha_{2}-0\right)}{f\left(\alpha_{2}\right)}+\operatorname{ctg} \Delta & \leqslant \frac{f\left(\alpha_{1}\right)}{f\left(\alpha_{2}\right)} \cdot \frac{1}{\sin \Delta} .
\end{aligned}
$$

Перемножая (15) и (16), получаем требуемое неравенство (14). Отметим, что перемножение корректно, так как левые части неравенств (15) и (16) не могут быть одновременно меньше нуля в силу (13). Лемма 1 доказана.

СлЕДСТВИЕ. Если $q\left(\alpha_{2}-0\right)=q\left(\alpha_{1}+0\right)=\gamma$ для некоторых чисел $\alpha_{1} u \alpha_{2}>\alpha_{1}$, то для всех $\alpha \in\left[\alpha_{1}, \alpha_{2}\right]$ имеем

$$
|\gamma-\alpha|<\frac{\pi}{2}, \quad f(\alpha)=A \cos (\gamma-\alpha),
$$

где $A$ - некоторая положительная постоянная.

Действительно, в силу монотонности $q(\alpha)$ получаем

$$
q(\alpha) \equiv \gamma, \quad \alpha_{1} \leqslant \alpha \leqslant \alpha_{2}
$$

Следовательно, $|\gamma-\alpha|<\pi / 2$ и $f^{\prime}(\alpha)=f(\alpha) \operatorname{tg}(\gamma-\alpha)$ для $\alpha \in\left[\alpha_{1}, \alpha_{2}\right]$, т.е. $f(\alpha)$ имеет вид $A \cos (\gamma-\alpha)$, где $A>0$, что и требовалось. 
Лемма 2. Пусть $f \in T$. Тогда

1) для любого $\gamma \in \mathbb{R}$ существует число $\alpha_{0} \in \mathbb{R}$ такое, что

$$
q\left(\alpha_{0}-0\right) \leqslant \gamma \leqslant q\left(\alpha_{0}+0\right)
$$

2) если $\gamma \in\left[q\left(\alpha_{1}-0\right), q\left(\alpha_{1}+0\right)\right]$ для некоторого $\alpha_{1} \in \mathbb{R}$, mo

$$
g_{m}(\gamma ; f)=\min _{\alpha \in \mathbb{R}} \frac{f(\alpha)}{|\cos (\gamma-\alpha)|}=\frac{f\left(\alpha_{1}\right)}{\cos \left(\gamma-\alpha_{1}\right)} .
$$

ДокАЗАТЕЛЬСТво. 1) Пусть $\gamma$ - фиксированное число, и пусть $\alpha_{0}$ - точка, в которой функция $f(\alpha) /|\cos (\gamma-\alpha)|$ достигает своего минимума по $\alpha$. Тогда $\cos \left(\gamma-\alpha_{0}\right) \neq 0$ и в силу $\pi$-периодичности $f(\alpha)$ можно считать, что $\left|\gamma-\alpha_{0}\right|<\pi / 2$.

Неотрицательная функция

$$
S(\alpha)=\ln f(\alpha)-\ln |\cos (\gamma-\alpha)|-\ln g_{m}(\gamma ; f)
$$

достигает при $\alpha=\alpha_{0}$ своего минимума. Поэтому $S^{\prime}\left(\alpha_{0}+0\right) \geqslant 0$ и $S^{\prime}\left(\alpha_{0}-0\right) \leqslant 0$, т.е.

$$
\frac{f^{\prime}\left(\alpha_{0}+0\right)}{f\left(\alpha_{0}\right)} \geqslant \operatorname{tg}\left(\gamma-\alpha_{0}\right), \quad \frac{f^{\prime}\left(\alpha_{0}-0\right)}{f\left(\alpha_{0}\right)} \leqslant \operatorname{tg}\left(\gamma-\alpha_{0}\right),
$$

что равносильно (17).

2) Если $\alpha_{1}=\alpha_{0}$, то утверждение 2) следует из доказательства части 1). Пусть $\alpha_{0}>\alpha_{1}$. Тогда в силу леммы 1 и условия 2 ) леммы 2

$$
\gamma \leqslant q\left(\alpha_{1}+0\right) \leqslant q\left(\alpha_{0}-0\right) .
$$

Но в силу (17) $\gamma \geqslant q\left(\alpha_{0}-0\right)$; следовательно,

$$
q\left(\alpha_{0}-0\right)=q\left(\alpha_{1}+0\right)=\gamma .
$$

Тогда $f(\alpha)=A \cos (\gamma-\alpha)$ при $\alpha \in\left[\alpha_{1}, \alpha_{0}\right]$ согласно следствию к лемме 1. Таким образом,

$$
g_{m}(\gamma ; f)=\frac{f\left(\alpha_{0}\right)}{\cos \left(\gamma-\alpha_{0}\right)}=\frac{f\left(\alpha_{1}\right)}{\cos \left(\gamma-\alpha_{1}\right)}=A,
$$

т.е. (18) доказано. Случай $\alpha_{0}<\alpha_{1}$ исследуется аналогично. Лемма 2 доказана.

Лемма 3. Если $f \in T$, то функиия $g_{m}(\gamma ; f)$ принадлежст классу $G$ и является решением уравнения (1).

ДокАЗАтЕльство. Непрерьвность и $\pi$-периодичность функции $g_{m}(\gamma ; f)$, а также свойства $g_{m}(\gamma ; f) \geqslant 0, g_{m}(\gamma ; f) \neq 0$ очевидны. Остается показать, что

$$
f(\alpha)=\max _{\gamma}|\cos (\gamma-\alpha)| g_{m}(\gamma ; f) .
$$

Из определения (5) функции $g_{m}(\gamma ; f)$ непосредственно следует, что для любых $\gamma$ и $\alpha \in \mathbb{R}$

$$
|\cos (\gamma-\alpha)| g_{m}(\gamma ; f) \leqslant f(\alpha) .
$$

Пусть теперь $\alpha=\alpha_{1}$ фиксировано, а $\gamma=q\left(\alpha_{1}+0\right)$. Согласно лемме 2

$$
\left|\cos \left(\gamma-\alpha_{1}\right)\right| g_{m}(\gamma ; f)=f\left(\alpha_{1}\right) .
$$

Очевидно, из (20) и (21) следует (19). Лемма 3 доказана. 
ДОКАЗАТЕЛЬСТВО ТЕОРЕМЫ 1 . Пусть решение $g(\gamma)$ уравнения (1) в классе $G$ существует, т.е. $g \in G$ и

$$
f(\alpha)=\max _{\gamma} V(\gamma, \alpha), \quad V(\gamma, \alpha)=g(\gamma)|\cos (\gamma-\alpha)|
$$

Из $\pi$-периодичности $|\cos x|$ сразу следует $\pi$-периодичность функции $f(\alpha)$.

Далее, функция $f(\alpha)$ должна быть строго положительной. Действительно, если $f(\alpha)=0$ для некоторой точки $\alpha$, то $g(\gamma)=0$ всюду за исключением точек $\gamma=\alpha+$ $\pi / 2+n \pi, n \in \mathbb{N}$. Тогда $g(\gamma) \equiv 0$ в силу непрерьвности, что противоречит условию $g \in G$.

Функция $|\cos (\gamma-\alpha)|$ является тригонометрически выпуклой по $\alpha$. Поэтому для любых $\alpha_{1}$ и $\alpha_{2}, 0<\alpha_{2}-\alpha_{1}<\pi$, и любом фиксированном $\gamma$

$$
V(\gamma, \alpha) \leqslant \frac{V\left(\gamma ; \alpha_{1}\right) \sin \left(\alpha_{2}-\alpha\right)+V\left(\gamma ; \alpha_{2}\right) \sin \left(\alpha-\alpha_{1}\right)}{\sin \left(\alpha_{2}-\alpha_{1}\right)}, \quad \alpha_{1} \leqslant \alpha \leqslant \alpha_{2}
$$

Беря от обеих частей максимум по $\gamma$, приходим к нужному неравенству (6) для функции $f(\alpha)$. Таким образом, необходимость условия $f \in T$ для разрешимости уравнения (1) в классе $G$ доказана.

Достаточность условия $f \in T$ для разрешимости и вторая часть теоремы 1 доказаны в лемме 3. Итак, теорема 1 доказана полностью.

ДоКАЗАТЕЛЬСТВо ТЕОРЕМЫ 2. В силу леммы 1 для различных $\alpha \in I$ множества $(q(\alpha-0), q(\alpha+0))$ представляют собой непересекающиеся между собой интервалы. Таким образом, для $f \in T$ множество

$$
X_{f}=\bigcup_{\alpha \in I}(q(\alpha-0), q(\alpha+0))
$$

является открытым, а множество $D_{f}=\mathbb{R} \backslash X_{f}$ является замкнутым. Отметим, что $D_{f}$ представляет собой область значений функций $q(\alpha \pm 0)$.

Если $f \in T$ и $f^{\prime}(\alpha)$ не имеет точек разрьва, то $D_{f}=\mathbb{R}$ и утверждение о единственности решения вытекает из следующей леммы.

ЛЕмма 4. Если $g$ и $g^{*}$ принадлежат классу $G$ и являются решениями уравнения (1) с одной и той же правой частью $f \in T$, mо $g(\gamma)=g^{*}(\gamma)$ для всех $\gamma \in D_{f}$.

ДокАЗАТЕЛЬСТво. Пусть $\gamma_{0} \in D_{f}$. Тогда либо $\gamma_{0}=q\left(\alpha_{0}+0\right)$, либо $\gamma_{0}=q\left(\alpha_{0}-0\right)$ для некоторого $\alpha_{0} \in \mathbb{R}$.

Предположим сначала, что $\alpha_{0} \notin I$. Множество $I$ не более чем счетно, поэтому в силу леммы 2 такие точки $\gamma_{0} \in D_{f}$ существуют. Пусть $\left|\cos \left(\gamma-\alpha_{0}\right)\right| g(\gamma)$ достигнет своего максимума в точке $\gamma_{1}$, а $\left|\cos \left(\gamma-\alpha_{0}\right)\right| g^{*}(\gamma)$ - в точке $\gamma_{2}$. Можно считать, что $\left|\gamma_{1}-\alpha_{0}\right|<\pi / 2$ и $\left|\gamma_{2}-\alpha_{0}\right|<\pi / 2$. Функции двух переменных

$$
\begin{aligned}
S(\gamma, \alpha) & =\ln f(\alpha)-\ln |\cos (\gamma-\alpha)|-\ln g(\gamma), \\
S^{*}(\gamma, \alpha) & =\ln f(\alpha)-\ln |\cos (\gamma-\alpha)|-\ln g^{*}(\gamma)
\end{aligned}
$$

достигают тогда своих минимумов соответственно в точках $\left(\gamma_{1}, \alpha_{0}\right)$ и $\left(\gamma_{2}, \alpha_{0}\right)$. Следовательно,

$$
\frac{\partial S}{\partial \alpha}\left(\gamma_{1}, \alpha_{0}\right)=0, \quad \frac{\partial S^{*}}{\partial \alpha}\left(\gamma_{2}, \alpha_{0}\right)=0
$$


что означает соответственно

$$
\frac{f^{\prime}\left(\alpha_{0}\right)}{f\left(\alpha_{0}\right)}=\operatorname{tg}\left(\gamma_{1}-\alpha_{0}\right), \quad \frac{f^{\prime}\left(\alpha_{0}\right)}{f\left(\alpha_{0}\right)}=\operatorname{tg}\left(\gamma_{2}-\alpha_{0}\right) .
$$

Отсюда получаем

$$
\gamma_{1}=\gamma_{2}=\gamma_{0}=q\left(\alpha_{0}\right)
$$

и с учетом леммы 2

$$
g\left(\gamma_{0}\right)=\frac{f\left(\alpha_{0}\right)}{\cos \left(\gamma_{0}-\alpha_{0}\right)}=g^{*}\left(\gamma_{0}\right)
$$

Случай $\alpha_{0} \in I$ получается предельным переходом. Действительно, пусть, например, $\gamma_{0}=q\left(\alpha_{0}+0\right)$. Так как $I$ не более чем счетно и $q(\alpha+0)$ непрерьвна справа, то можно указать последовательность $\left\{\alpha_{n}\right\}$ такую, что $\alpha_{n} \notin I, \alpha_{n}>\alpha, \lim \alpha_{n}=\alpha, \gamma_{n}=q\left(\alpha_{n}\right)$, $\lim q\left(\alpha_{n}\right)=q(\alpha+0)=\gamma_{0}$. По доказанному вьше

$$
g\left(\gamma_{n}\right)=\frac{f\left(\alpha_{n}\right)}{\cos \left(\gamma_{n}-\alpha_{n}\right)}=g^{*}\left(\gamma_{n}\right)
$$

Отсюда и следует требуемое равенство в силу непрерьвности функций $g(\gamma), g^{*}(\gamma)$ и $f(\alpha)$. Лемма 4 доказана.

Докажем теперь вторую часть теоремы 2. Пусть $g \in G$ и $g(\gamma)$ является решением уравнения (1). Тогда для любых $\gamma$ и $\alpha \in \mathbb{R}$

$$
g(\gamma) \leqslant \frac{f(\alpha)}{|\cos (\gamma-\alpha)|}
$$

в частности,

$$
g(\gamma) \leqslant \min _{\alpha} \frac{f(\alpha)}{|\cos (\gamma-\alpha)|}=g_{m}(\gamma ; f) .
$$

Кроме того, в силу лемм 3 и $4 g(\gamma)=g_{m}(\gamma ; f)$, если $\gamma \in \mathbb{R} \backslash X_{f}$. Тем самьм, доказана необходимость условий (7).

Докажем достаточность. Пусть $g(\gamma) \in G$ и вьполняются условия (7). Тогда для любого $\gamma$ и $\alpha \in \mathbb{R}$

$$
g(\gamma)|\cos (\gamma-\alpha)| \leqslant g_{m}(\gamma ; f)|\cos (\gamma-\alpha)| \leqslant f(\alpha)
$$

С другой стороны, для любого $\alpha_{1} \in \mathbb{R}$ положим $\gamma_{1}=q\left(\alpha_{1}+0\right)$. Тогда $\gamma_{1} \in D_{f}=\mathbb{R} \backslash X_{f}$, т.е. $g\left(\gamma_{1}\right)=g_{m}\left(\gamma_{1} ; f\right)$. По лемме 2

$$
g\left(\gamma_{1}\right)\left|\cos \left(\gamma_{1}-\alpha_{1}\right)\right|=g_{m}\left(\gamma_{1} ; f\right)\left|\cos \left(\gamma_{1}-\alpha_{1}\right)\right|=f\left(\alpha_{1}\right)
$$

т.е. $g(\gamma)$ является решением уравнения (1). Таким образом, теорема 2 доказана. 
ДокАЗАТЕЛЬСТво ТЕОРЕмЫ 3. Докажем сначала, что для всех $\gamma \in\left[q\left(\alpha_{1}-0\right)\right.$, $\left.q\left(\alpha_{2}+0\right)\right]$ имеет место равенство

$$
\min _{\alpha_{1} \leqslant \alpha \leqslant \alpha_{2}} \frac{f(\alpha)}{|\cos (\gamma-\alpha)|}=\min _{\alpha \in \mathbb{R}} \frac{f(\alpha)}{|\cos (\gamma-\alpha)|}=g_{m}(\gamma ; f) .
$$

По лемме 2 для фиксированного

$$
\gamma \in\left[q\left(\alpha_{1}-0\right), q\left(\alpha_{2}+0\right)\right]
$$

существует $\alpha_{0}$ такое, что

$$
q\left(\alpha_{0}-0\right) \leqslant \gamma \leqslant q\left(\alpha_{0}+0\right) .
$$

Если $\alpha_{0} \in\left[\alpha_{1}, \alpha_{2}\right]$, то (22) непосредственно следует из (18).

Пусть $\alpha_{0}<\alpha_{1}$. Тогда, с одной стороны, в силу (23) и монотонности $q(\alpha)$ имеем

$$
q\left(\alpha_{0}+0\right) \leqslant q\left(\alpha_{1}-0\right) \leqslant \gamma
$$

a, с другой стороны, в силу (24) имеем $q\left(\alpha_{0}+0\right) \geqslant \gamma$. Следовательно, $q\left(\alpha_{1}-0\right)=\gamma$ и на основании (18) минимум функции $f(\alpha) /|\cos (\alpha-\gamma)|$ по $\alpha \in \mathbb{R}$ достигается при $\alpha=\alpha_{1}$, т.е. снова (22) вытекает из (18). Аналогично исследуется случай $\alpha_{0}>\alpha_{2}$.

Итак, равенство (22) для $\gamma \in\left[q\left(\alpha_{1}-0\right), q\left(\alpha_{2}+0\right)\right]$ обосновано. Доказательство теоремы 3 завершается теперь следующим образом.

Пусть $f(\alpha) \geqslant f^{*}(\alpha)$ при $\alpha \in\left[\alpha_{1}, \alpha_{2}\right]$, причем $f$ и $f^{*} \in T$. Тогда имеет место неравенство

$$
\min _{\alpha_{1} \leqslant \alpha \leqslant \alpha_{2}} \frac{f(\alpha)}{|\cos (\gamma-\alpha)|} \geqslant \min _{\alpha_{1} \leqslant \alpha \leqslant \alpha_{2}} \frac{f^{*}(\alpha)}{|\cos (\gamma-\alpha)|}, \quad \gamma \in \mathbb{R} .
$$

Из (22), (25) и очевидного неравенства

$$
\min _{\alpha_{1} \leqslant \alpha \leqslant \alpha_{2}} \frac{f^{*}(\alpha)}{|\cos (\gamma-\alpha)|} \geqslant \min _{\alpha \in \mathbb{R}} \frac{f^{*}(\alpha)}{|\cos (\gamma-\alpha)|}=g_{m}\left(\gamma ; f^{*}\right), \quad \gamma \in \mathbb{R}
$$

следует доказьваемоенеравенство $g_{m}(\gamma, f) \geqslant g_{m}\left(\gamma, f^{*}\right)$ для всех $\gamma \in\left[q\left(\alpha_{1}-0\right), q\left(\alpha_{2}+0\right)\right]$. Теорема 3 доказана.

ЗАМЕЧАнИЕ 1 . Из (5) следует, что для $f(\alpha)>0$

$$
\frac{1}{g_{m}(\gamma ; f)}=\max _{\alpha \in \mathbb{R}} \frac{|\cos (\gamma-\alpha)|}{f(\alpha)}, \quad \gamma \in \mathbb{R}
$$

В силу тригонометрической вьпуклости $|\cos x|$ получаем, что

$$
\frac{1}{g_{m}(\gamma ; f)} \in T
$$

Следовательно, максимальное решение $g_{m}(\gamma ; f)>0$ для всех $\gamma \in \mathbb{R}$ и $g_{m}(\gamma ; f)$ имеет непрерьвную почти всюду производную. 
ЗАмЕчАнИЕ 2. Класс функций $G$ может быть расширен посредством исключения условий $g(\gamma) \geqslant 0$ и $g(\gamma) \not \equiv 0$. При этом к классу правых частей $T$ добавляется всего одна функция $f(\alpha) \equiv 0$.

В самом деле, если $f(\alpha)=0$ при некотором $\alpha$, то в силу непрерьвности $g(\gamma)$ получаем $g(\gamma) \leqslant 0$. Следовательно, $f(\alpha) \equiv 0$, так как $\cos (\gamma-\alpha)=0$ при любом $\gamma=\alpha+\pi / 2+n \pi$, $n \in \mathbb{N}$. По той же причине не может быть $f(\alpha)<0$ при каком-либо $\alpha$.

Таким образом, если допустить, что $f(\alpha) \equiv 0$, то решением уравнения (1) будет любая неположительная функция $g(\gamma)$. Если же $f(\alpha)>0$ и допускается, что $g(\gamma)<0$, то множество непрерьвных, $\pi$-периодических решений полностью описьвается условиями (7), что следует из способа доказательства теоремы 2.

4. Заключение. Мы уже отметили, что уравнение (1) возникло в прикладной задаче. Отвлекаясь от физического смысла задачи, можно попытаться рассмотреть уравнения более общего вида

$$
\max _{\gamma \in \mathbb{R}} g(\gamma) K(\gamma-\alpha)=f(\alpha)
$$

Пусть $K(\alpha)$ и $f(\alpha)$ - периодические, непрерывные функции с одним и тем же периодом. Предположим, что $K(\alpha) \geqslant 0$ и $K(\alpha)=0$ лишь в конечном числе точек на периоде. Рассмотрим функцию

$$
g_{m}(\gamma ; f)=\min _{\alpha \in \mathbb{R}} \frac{f(\alpha)}{K(\gamma-\alpha)} .
$$

Обозначим через $L(\gamma)$ множество точек, в которых функция $f(\alpha) / K(\gamma-\alpha)$ достигает своего минимума по $\alpha$ при фиксированном $\gamma$, и пусть

$$
M=\bigcup_{\gamma \in \mathbb{R}} L(\gamma)
$$

Ясно, что если $g(\gamma)$ есть решение уравнения $(26)$, то $g(\gamma) \leqslant g_{m}(\gamma ; f)($ см. доказательство необходимости условий (7)). Кроме того, при любых $\gamma$ и $\alpha$

$$
g_{m}(\gamma ; f) K(\gamma-\alpha) \leqslant f(\alpha) .
$$

Равенство в (27) возможно тогда и только тогда, когда $\alpha \in M$. Отсюда следует, что функция $g_{m}(\gamma ; f)$ будет решением уравнения $(26)$, только если $M=\mathbb{R}$.

$\mathrm{B}$ данной статье мы доказали, что если $K(\alpha)=|\cos (\alpha)|$, то $M=\mathbb{R}$. Очевидно, что свойства косинуса не уникальны и что существует класс ядер, для которых вьполняется указанное условие. Вопрос об описании этого класса, а также класса соответствующих правых частей $f(\alpha)$ может быть предметом дальнейших исследований.

\section{СПИСОК ЦИТИРОВАННОЙ ЛИТЕРАТУРЫ}

[1] Авхадиев Ф.Г., Маклаков Д. В. Критерий разрешимости задачи построения профилей по заданной кавитационной диаграмме // Изв. вузов. Матем. 1994. № 7. С. 3-12.

[2] Авхадиев Ф.Г., Маклаков Д. В. Аналитический метод построения гидропрофилей по заданной кавитационной диаграмме // Докл. РАН. 1995. Т. 343. №2. С. 195-197.

[3] Avhadiev F. G., Maklakov D. V. A theory of pressure envelope for hydrofoils // J. Ship Technology Research. 1995. V. 42. № 2. P. 81-102.

[4] Левин Б. Я. Распределение корней целых функций. М.: ГИТТЛ, 1956.

[5] Харди Г. Г., Литтльвуд Дж. Е., Полиа Г. Неравенства. М.: ИЛ, 1948.

(Ф. Г. Авхадиев) НИИ математики и механики им. Н.Г. Чеботарева

Поступило при Казанском государственном университете

03.04 .2000

(Д. В. Маклаков) Казанская государственная архитектурно-строительная академия

E-mail: Farit.Avhadiev@ksu.ru, Dmitri.Maklakov@ksu.ru 